\title{
Riemannian \\ Manifolds of \\ Conullity Two
}


This page is intentionally left blank 


\section{Riemannian}

\section{Manifolds of}

\section{Conullity Two}

\section{Eric Boeckx}

Katholieke Universiteit Leuven, Belgium

Oldřich Kowalski

Charles University, Czech Republic

\section{Lieven Vanhecke}

Katholieke Universiteit Leuven, Belgium 


\section{Published by}

World Scientific Publishing Co. Pte. Ltd.

P O Box 128, Farrer Road, Singapore 912805

USA office: Suite 1B, 1060 Main Street, River Edge, NJ 07661

UK office: 57 Shelton Street, Covent Garden, London WC2H 9HE

\section{British Library Cataloguing-in-Publication Data}

A catalogue record for this book is available from the British Library.

\section{RIEMANNIAN MANIFOLDS OF CONULLITY TWO}

Copyright 191996 by World Scientific Publishing Co. Pte. Ltd.

All rights reserved. This book, or parts thereof, may not be reproduced in any form or by any means, electronic or mechanical, including photocopying, recording or any information storage and retrieval system now known or to be invented, without written permission from the Publisher.

For photocopying of material in this volume, please pay a copying fee through the Copyright Clearance Center, Inc., 222 Rosewood Drive, Danvers, MA 01923, USA. In this case permission to photocopy is not required from the publisher.

ISBN 981-02-2768-X

This book is printed on acid-free paper.

Printed in Singapore by Uto-Print 


\section{Contents}

Introduction $\quad$ xi

1 Definition of semi-symmetric spaces and early development $\quad 1$

1.1 Notational conventions . . . . . . . . . . . . . . . . . . 1

1.2 The Riemann curvature tensor as equivariant map . . . 2

1.3 Definition of a semi-symmetric space . . . . . . . . . 4

1.4 The extrinsic analogue: symmetric and semi-parallel submanifolds ................ 5

1.5 Early development . . . . . . . . . . . . 7

2 Local structure of semi-symmetric spaces 11

2.1 The local structure theorem . . . . . . . . . . . . 11

2.2 Semi-symmetric cones . . . . . . . . . . . 16

2.2 .1 The real cones . . . . . . . . . . 17

2.2 .2 The Kählerian cones . . . . . . . . . . . . . . . 19

2.3 Riemannian manifolds of conullity two . . . . . . . . . 19

3 Explicit treatment of foliated semi-symmetric spaces 23

3.1 A canonical form for the metrics and the basic system of partial differential equations . . . . . . . . . 23

3.2 First integrals . . . . . . . . . . . . . . . . 32

3.3 Two possible simplifications . . . . . . . . . . . 34

$3.3 .1 \mathbf{A}^{2}$ has a special form . . . . . . . . . . 34

3.3.2 The functions $\mathbf{G}^{\alpha}$ are identically zero . . . . 38 
4 Curvature homogeneous semi-symmetric spaces $\mathbf{4 3}$

4.1 Curvature homogeneous spaces . . . . . . . . . . 43

4.2 Semi-symmetric curvature homogeneous spaces . . . . . 45

4.2.1 Conditions for local symmetry . . . . . . . . 45

4.2 .2 Local structure . . . . . . . . . . . . . . . 48

4.2.3 Sekigawa's example and its extensions . . . . . . 51

4.3 Complete local classification . . . . . . . . . . . . 54

4.3.1 Technical apparatus . . . . . . . . . 54

4.3.2 The germs of metrics . . . . . . . . . . 60

4.4 An extension : spaces with constant scalar curvature along the leaves ................. . . . 65

4.5 An application: Einstein-like semi-symmetric spaces . . 67

5 Asymptotic foliations and algebraic rank 73

5.1 Asymptotic foliations . . . . . . . . . . . 73

5.2 Algebraic rank . . . . . . . . . . . . 80

6 Three-dimensional Riemannian manifolds of conullity two $\quad 85$

6.1 Three-dimensional foliated semi-symmetric spaces . . . 85

6.2 Riemannian invariants and isometries . . . . . . 88

6.3 Classification of three-dimensional asymptotically foliated semi-symmetric spaces . . . . . . . . . . . 92

6.3.1 Planarly foliated semi-symmetric spaces . . . . . 92

6.3.2 Parabolically foliated semi-symmetric spaces . . 94

6.3.3 Orthogonally hyperbolically foliated semi-symmetric spaces . . . . . . . . . . . . 100

6.3.4 Non-orthogonally hyperbolically foliated semi-symmetric spaces . . . . . . . . . . . . 103

7 Asymptotically foliated semi-symmetric spaces $\quad 109$

7.1 Classification of asymptotically foliated semi-symmetric spaces with algebraic rank one . . . . . . . . . 110

7.1.1 Planarly foliated semi-symmetric spaces . . . . . 110

7.1.2 Parabolically foliated semi-symmetric spaces . . 111

7.1.3 Orthogonally hyperbolically foliated semi-symmetric spaces . 
7.1.4 Non-orthogonally hyperbolically foliated semi-symmetric spaces . . . . . . . . . . . . . . . 119

7.2 Examples of asymptotically foliated spaces with higher algebraic rank . . . . . . . . . . . . . 125

7.2.1 Hyperbolically foliated semi-symmetric spaces with algebraic rank two . . . . . . . . . 125

7.2.2 Parabolically foliated semi-symmetric spaces with algebraic rank two . . . . . . . . . . 127

7.2.3 Parabolically foliated semi-symmetric spaces with algebraic rank three . . . . . . . . . . 128

7.3 An application: semi-symmetric $\mathfrak{P}$-spaces . . . . . . 130

8 Elliptic semi-symmetric spaces $\quad 137$

8.1 General construction for elliptic semi-symmetric spaces with algebraic rank one . . . . . . . . . . 137

8.2 Examples of elliptic semi-symmetric spaces with higher algebraic rank . . . . . . . . . . . . . . 144

8.2.1 Elliptic semi-symmetric spaces with algebraic rank equal to two or three . . . . . . . . . . . 145

8.2.2 Elliptic semi-symmetric spaces with algebraic rank equal to two, three or four . . . . . . . . . 151

9 Complete foliated semi-symmetric spaces

9.1 Three different classes of complete foliated semi-symmetric spaces . . . . . . . . . . . . . . . 159

9.2 Semi-symmetric spaces of hyperbolic type (in the sense of Szabó) . . . . . . . . . . . . . . 162

9.2.1 Complete semi-symmetric hypersurfaces in Euclidean spaces . . . . . . . . . . . . . . 163

9.2.2 Szabó's construction for complete semisymmetric hypersurfaces of hyperbolic type . . . 166

9.2 .3 New examples . . . . . . . . . . . . 170

9.3 Semi-symmetric spaces of parabolic type (in the sense of Szabó) . . . . . . . . . . . . . 175

9.4 The relation between the two terminologies . . . . . 177 
10 Application : local rigidity problems for hypersurfaces with type number two in $\mathbb{R}^{4}$

10.1 Hypersurfaces of Euclidean spaces . . . . . . . . . 182

10.2 The basic system of partial differential equations . . . . 185

10.3 Three-dimensional foliated semi-symmetric spaces as hypersurfaces in $\mathbb{R}^{4} \ldots \ldots \ldots$. . . . . . . 188

10.3.1 Planarly foliated semi-symmetric spaces . . . . 188

10.3.2 Parabolically foliated semi-symmetric spaces . . 190

10.3.3 Orthogonally hyperbolically foliated semi-symmetric spaces . . . . . . . . . . . 198

10.4 Ruled hypersurfaces in $\mathbb{R}^{4} \ldots \ldots . \ldots 207$

11 Three-dimensional Riemannian manifolds of c-conullity two

11.1 Introduction . . . . . . . . . . . . . . . . 219

11.2 The basic system of partial differential equations . . . . 223

11.3 Riemannian invariants . . . . . . . . . . . . . . 227

11.4 Asymptotic foliations and four types of spaces . . . . . 229

11.5 The explicit classification of asymptotically foliated spaces of hyperbolic type . . . . . . . . . . . 231

11.6 The explicit classification of asymptotically foliated spaces of elliptic type . . . . . . . . . . . . . . . 241

11.7 Concluding remarks . . . . . . . . . . . . . . 249

12 More about curvature homogeneous spaces 251

12.1 Introduction . . . . . . . . . . . . . . 251

12.2 Examples of curvature homogeneous spaces of non-symmetric type . . . . . . . . . . . 253

12.2.1 Isoparametric hypersurfaces in spheres . . . . . 253

12.2.2 Curvature homogeneous hypersurfaces in real space forms . . . . . . . . . . . 254

12.2.3 Curvature homogeneous spaces on two-step solvable Lie groups . . . . . . . . . . . 255

12.3 Three-dimensional curvature homogeneous spaces . . . . 259 12.3.1 The case $\rho_{1}=\rho_{2} \neq \rho_{3} \ldots \ldots \ldots$. . . . . . . . . . . .

12.3.2 The case of distinct $\rho_{1}, \rho_{2}, \rho_{3} \ldots \ldots 264$

12.3.3 Main existence theorem . . . . . . . . . . . . 272 
12.4 Curvature homogeneity in higher dimensions . . . . 273

12.5 Extensions . . . . . . . . . . . . . . . 276

12.5.1 The pseudo-Riemannian case . . . . . . . . . 276

12.5.2 The affine case . . . . . . . . . . . . . 277

12.6 The Singer theorem and local homogeneity . . . . . 278

Bibliography

283

Index

299 
This page is intentionally left blank 


\section{Introduction}

In several fields of science, and in particular in differential geometry, Riemannian symmetric spaces play a prominent role. The analytic condition expressing (local) symmetry, $D R=0$, implies the local homogeneity and hence, the Riemann curvature tensor $R$ of a (locally) symmetric space is the "same" at each point. Moreover, $R$ is not arbitrary: E. Cartan proved that an algebraic curvature tensor $K$ belongs to some-symmetric space if and only if it satisfies the additional condition $K(X, Y) \cdot K=0$. It is then a natural step to define a (broader) class of Riemannian manifolds by requiring that they satisfy the curvature condition $R(X, Y) \cdot R=0$. Riemannian manifolds satisfying this condition are called semi-symmetric spaces. Actually, the above condition means that, at every point $p$ of a semi-symmetric space $(M, g)$, the Riemann curvature tensor $R_{p}$ belongs to some symmetric space, but this symmetric space can change from point to point. For example, it is easy to see that all two-dimensional Riemannian manifolds are semi-symmetric.

The first mathematician who paid attention to the class of semisymmetric spaces was E. Cartan, in connection with his fundamental research on (locally) symmetric spaces. Many other mathematicians continued this study (see Chapter 1). A major and fruitful impulse was provided by K. Nomizu in 1968: in [98], he conjectures that all complete, irreducible semi-symmetric manifolds of dimension greater than or equal to three are locally symmetric. His conjecture was refuted in 1972 by $\mathrm{H}$. Takagi ([141]) who constructed a hypersurface in $\mathbb{R}^{4}$ which provided the first counterexample, and by K. Sekigawa ([119]) who published counterexamples of arbitrary dimensions. These 
results raised the problem of classifying all semi-symmetric spaces.

Between 1982 and 1985, Z. I. Szabó published three fundamental papers on the subject: in [138], he gives the full local classification of Riemannian semi-symmetric spaces; [139] treats complete semisymmetric spaces and [140] deals with the classification and construction of complete hypersurfaces of Euclidean spaces which are semisymmetric with respect to the induced metric.

The local structure theorem in [138] (see Theorem 2.9) says that every locally irreducible semi-symmetric space falls into one of the following three classes (the terminology is due to [59]) :

1) the "trivial" class: all locally symmetric spaces and all twodimensional Riemannian surfaces;

2) the "exceptional" class: elliptic cones, hyperbolic cones, Euclidean cones and Kählerian cones;

3) the "typical" class: Riemannian spaces foliated by Euclidean leaves of codimension two.

Whereas the "trivial" semi-symmetric manifolds are well-understood and the "exceptional" ones are described and constructed explicitly in [138] and [139], the foliated semi-symmetric spaces are given here only implicitly, i.e., Szabó does not provide explicit expressions for the metric of such spaces, but he only gives an integrable system of nonlinear partial differential equations and the exact number of solutions: the solutions in dimension $n$ depend in general on $(n-2)(n+3) / 2+4$ arbitrary functions of two variables and $(n-2)(n+3) / 2$ arbritrary functions of one variable. In [138], however, no explicit solutions are given. As far as we know, the first explicit examples depending essentially on one arbitrary function of two variables were constructed by F. Tricerri, the second and the third author in [73], [74] as generalisations of two examples of K. Sekigawa ([124]).

In 1991, the second author made a systematic study of three-dimensional foliated semi-symmetric spaces ([59]). He deduces a system of partial differential equations equivalent to that of Szabó, but by a different method and in a different form. Then he introduces the geometric notion of an "asymptotic foliation" (whose leaves are of 
codimension one) and divides the class of semi-symmetric manifolds into four subclasses: the planar, the hyperbolic, the parabolic and the elliptic spaces, depending on the number of asymptotic foliations they admit. Further, he succeeds in solving the basic system of partial differential equations to obtain explicit expressions for the metrics of such spaces, except for the elliptic case, where only a quasi-explicit formula is given. Finally, he proves that the local isometry classes of these metrics can be parametrised by three arbitrary functions of two variables, which is much less than the number of arbitrary functions derived formally by Szabó (his formula gives seven functions of two variables for $n=3$ ).

In this book, we will study foliated semi-symmetric spaces (in fact, Riemannian manifolds of conullity two) of arbitrary dimensions. We derive the basic system of partial differential equations and define "asymptotic foliations" also in higher dimensions. Moreover, we introduce a new geometric invariant for a foliated semi-symmetric space, the "algebraic rank". Using these two concepts, we give various classifications and examples. We apply our results to the theory of hypersurfaces with type number two in the Euclidean space $\mathbb{R}^{4}$. Then we extend, in dimension three, our results to a broader class of Riemannian manifolds which are "elliptic" and "hyperbolic" analogues of spaces of conullity two. Finally, we add a special expository (and upto-date) survey on curvature homogeneous spaces. This last chapter is related to the previous topic rather by the similarity of methods than by contents.

The contents of the subsequent chapters is as follows. In Chapter 1, we give a rigourous definition for semi-symmetric spaces and we inform briefly about the extrinsic analogue of these spaces (called semi-parallel submanifolds). In the last section of this chapter, we give a concise overview of the early research about semi-symmetric spaces. This survey is certainly not exhaustive. We refer to the quoted papers for more detailed information and further references.

Chapter 2 is devoted to the fundamental local structure theorem by Z. I. Szabó. We indicate the major steps in his reasoning and take a closer look at the different irreducible factors in which a general semi-symmetric space can be decomposed (locally). 
In Chapter 3, we start the (local) investigation of foliated semisymmetric spaces of arbitrary dimension. Generalising the procedure known for the three-dimensional case, we first derive the basic system of partial differential equations for our problem. Next, we find some first integrals for this system and present two simplifications of the problem, which will both be useful in what follows: the first one already in Chapter 4, the second one throughout Chapter 5 to Chapter 9 .

Chapter 4 deals with a special subclass of semi-symmetric spaces, namely those for which the curvature tensor is the same at each point, the so-called curvature homogeneous semi-symmetric spaces. Using the local structure theorem by Szabó, we reduce the classification problem to the study of foliated semi-symmetric spaces with constant scalar curvature. Using the techniques developed in Chapter 3, we determine an explicit formula for the local metrics of these spaces (Theorem 4.20). This allows to describe all non-symmetric curvature homogeneous spaces having the same curvature tensor as a fixed symmetric space. As an application of the results of this chapter, we show that every Einsteinlike semi-symmetric space is locally symmetric (Theorem 4.22) and we give some immediate consequences of this result.

In Chapter 5, we introduce the notion of an asymptotic foliation in arbitrary dimensions (as a codimension one foliation) and we define planar, hyperbolic, parabolic and elliptic semi-symmetric spaces according to the number of asymptotic foliations they admit (infinitely many, two, one or none, respectively). Moreover, we show that the existence of an asymptotic foliation has strong implications on the form of the metric (Theorem 5.3), which simplifies considerably the basic system of partial differential equations. Further, we define the geometric notion of algebraic rank for a foliated semi-symmetric space as an integer $r \in\{0,1,2,3,4\}$. We then prove that the case $r=0$ corresponds to a local product space $M^{2} \times \mathbb{R}^{n}$ (Proposition 5.5) and we determine the possible values of the algebraic rank for a planar, hyperbolic, parabolic and elliptic semi-symmetric space (Proposition 5.6).

Chapter 6 is devoted to the study of three-dimensional, non-elliptic, foliated semi-symmetric spaces. The explicit formulas from [59] for "generic" cases are derived here once again, using the previous general 
theory. But, besides that, all non-generic cases are also classified. The last material is based on the Doctoral Thesis by V. Hájková ([49]).

Chapter 7 contains a closer study of asymptotically foliated semisymmetric spaces, i.e., non-elliptic foliated semi-symmetric spaces. For the case of algebraic rank equal to one, we are able to give a complete local classification by explicit metrics. The procedure is rather technical and consists mainly in applying the appropriate coordinate transformations. In the second section of this chapter, we then give families of examples having higher algebraic rank and covering all possibilities. (We mention that spaces of higher algebraic rank, as well as non-trivial planar spaces and hyperbolic spaces, are never complete.)

Chapter 8 deals with the remaining class of elliptic foliated semisymmetric spaces. We present a general construction for these spaces when the algebraic rank is one. In particular, we show (as a local property) that an irreducible elliptic space of algebraic rank equal to one must be three-dimensional. Our treatment of these spaces is more uniform than that presented in [59]. We also give families of explicit examples of elliptic semi-symmetric spaces having higher algebraic rank and covering all possibilities.

In Chapter 9, we focus our attention on complete foliated semisymmetric spaces. These have been studied in detail by Szabó in [139]. The first section of this chapter is therefore devoted to a short survey of his work, including a division of these spaces into three different types: trivial, parabolic and hyperbolic ones. (This terminology is different from that used in Chapters 5-8.) The spaces of trivial type are all local product spaces. As concerns the spaces of hyperbolic type, only one explicit example of this type was known until recently, namely the counterexample to Nomizu's conjecture by Takagi ([141]). This is realised as a hypersurface in $\mathbb{R}^{4}$. In order to find other examples, we first review Szabó's work on complete semi-symmetric hypersurfaces in Euclidean spaces ([140]). He classifies these hypersurfaces and describes general constructions for such spaces. Applying his procedure, we then extend Takagi's example to a whole family parametrised by holomorphic functions defined on the whole complex plane (Theorem 9.12). For the spaces of parabolic type, we prove an alternative geometric characterisation (Theorem 9.13) and, using this, we deduce 
that the counterexamples to Nomizu's conjecture by Sekigawa ([119]) belong to this class. Finally, we clarify the relations between the spaces of trivial, parabolic and hyperbolic type in the sense of Szabó and the four classes of foliated semi-symmetric spaces (planar, hyperbolic, parabolic and elliptic) defined in Chapter 5.

In Chapter 10, we use the three-dimensional classification of Chapter 6 for the construction of hypersurfaces with type number two in $\mathbb{R}^{4}$. We obtain all possible situations anticipated by E. Cartan ([21]) as concerns the number of isometric deformations. In particular, by the embedding of certain "orthogonally hyperbolic spaces" one proves the existence of hypersurfaces admitting exactly one non-trivial isometric deformation. This result (belonging to V. Hájková ([49])) seems to be the first example of such a situation. (E. Cartan never proved the actual existence of these hypersurfaces. M. Dajczer, L. Florit and R. Tojeiro constructed such examples only very recently ([29]).) The last section presents the intrinsic classification of all ruled hypersurfaces in $\mathbb{R}^{4}$. (One obtains four different types of metrics, all written in explicit form.) Ruled hypersurfaces correspond to some "singular cases" of the classification from Chapter 6.

Chapter 11 is devoted to Riemannian manifolds of conullity two modulo some curvature tensor of constant sectional curvature $c$. The basic properties of foliated semi-symmetric spaces remain valid for these new classes of Riemannian manifolds. We restrict ourselves to the three-dimensional case and we give the classification of all generic asymptotically foliated spaces. The explicit formulas which we obtain are complete analogues of the corresponding formulas from Chapter 6 . Examples of such spaces are hypersurfaces with type number two in $S^{4}$ and $\mathbb{H}^{4}$. This extended class of spaces (including the case $c=0$ ) coincides, in dimension three, with the so-called "pseudo-symmetric spaces of constant type". A fair credit for this chapter is given to M. Sekizawa.

Chapter 12 is of an expository nature and is devoted to the recent results on curvature homogeneous spaces of non-symmetric type. (Those of symmetric type are treated in Chapter 4.) It includes a number of interesting isolated examples and also entire families of examples. The classification problem is solved, at least partially, in 
dimension three. A basic theorem says that every algebraic curvature tensor in dimension three can be realised on a curvature homogeneous space (but not always on a locally homogeneous one). Moreover, if the prescribed curvature tensor is not of constant sectional curvature, then there is an infinite-dimensional space of examples of a given type which are not locally homogeneous. The topic treated in this chapter was initiated in 1960 by I. M. Singer ([131]) and there are still numerous open problems left for the readers, including the so-called Gromov conjecture. The chapter is dedicated to the memory of our friend and collaborator F. Tricerri. He contributed a lot to this field of Riemannian geometry and he originally planned to write a joint monograph with the last two authors about curvature homogeneous spaces. But he could never realise this idea because of his tragic death, together with his entire family, in an airplane crash at Mingdu in China on June 6, 1994.

\section{Acknowledgments}

It is a pleasure to thank our colleagues and friends for their contributions to our work in general, and to this book in particular. We also take this opportunity to express our gratitude to the Charles University at Prague, the Katholieke Universiteit Leuven at Leuven and the University of Illinois at Urbana-Champaign for their hospitality and continued support. Thanks are also due to the Grant Agency of the Czech Republic (projects 201/93/0469, 201/96/0227), the National Fund for Scientific Research (NFWO) Belgium and the Belgian American Educational Foundation for their financial support during our research and the preparation of this book.

Urbana-Champaign, Prague, Leuven May 1996 